\title{
DECISIONS AND ACTIONS OF DISTRACTED DRIVERS AT THE ONSET OF YELLOW LIGHTS
}

\begin{abstract}
Driving on an approach to a signalized intersection while distracted is relatively risky, as potential vehicular conflicts and resulting angle collisions tend to be relatively more severe compared to other locations. Given the prevalence and importance of this particular scenario, the objective of this study was to examine the decisions and actions of distracted drivers during the onset of yellow lights. Driving simulator data were obtained from a sample of 69 drivers under baseline and handheld cell phone conditions at the University of Iowa National Advanced Driving Simulator. Explanatory variables included age, gender, cell phone use, distance to stop-line, and speed. Although there is extensive research on drivers' responses to yellow traffic signals, the examinations have been conducted from a traditional regression-based approach, which do not necessary provide the underlying relations and patterns among the sampled data. In this paper, we exploit the benefits of both classical statistical inference and data mining techniques to identify the a priori relationships among main effects, non-linearities, and interaction effects. Results suggest that the probability of yellow light running increases with the increase in driving speed at the onset of yellow. Both young (18-25 years) and middle-aged (30-45 years) drivers reveal reduced propensity for yellow light running whilst distracted across the entire speed range, exhibiting possible risk compensation during this critical driving situation. The propensity for yellow light running for both distracted male and female older (50-60 years) drivers is significantly higher. Driver experience captured by age interacts with distraction, resulting in their combined effect having slower physiological response and being distracted particularly risky.
\end{abstract}

Key words: Distracted driving; Mobile phone; Motion-based driving simulator; Risk compensation; Yellow light; Driver behavior 


\section{Introduction}

\subsection{Distracted Driving}

Driver distraction is a significant and growing road safety issue worldwide. Statistics reveal that about 3,328 people were killed and an additional 421,000 people were injured in distracted driving related motor vehicle crashes on U.S. roadways in 2012 (NHTSA, 2014). While cell phone use has become ubiquitous in modern society, road traffic crashes related to cell phone distractions have been on the rise (WHO, 2011). In 2012, cell phone distraction alone was associated with 415 fatalities and another 28,000 injuries on U.S. roads (NHTSA, 2014). Redelmeier and Tibshirani (1997) indicated that distraction due to conversing on a cell phone while driving increased the crash risk by as much as four folds. In 2010, the traffic police of Queensland, Australia fined about 30,780 drivers for talking or texting on mobile phones while driving, indicating the spread of cell phone usage (Ironside, 2011). Making matters worse, distracted driving tends to be more prevalent among younger and less experienced drivers. An Australian study reported that among the 2400 driver distraction related incidents in New South Wales, young drivers had the highest frequency of cell phone use-related injurious crashes (Lam, 2002).

Prior research has documented a variety of performance measures that have been impacted by the distracting effects of a cell phone. Burns et al. (2002) reported that speed control and reaction times of drivers were more influenced by a cell phone use than by having a blood alcohol level at the limit of $8 \%$. A recent study reported that cognitive distraction significantly impairs the reaction time of young drivers in response to a traffic event that originates within the driver's peripheral vision (Haque \& Washington, 2014a). Cell phone distraction has also been reported influencing drivers to have higher variation in accelerator pedal position, drive more slowly with greater speed variation, and report a higher workload (e.g., Rakauskas, Gugerty, \& Ward, 2004). Tornos and Bolling (2006) reported a risk compensation behavior of distracted drivers where drivers tend to reduce their speed while talking on a phone. Dula et al. (2011) showed that the percent of time spent speeding and the number of centre line crossings were significantly higher among drivers engaged in different types of conversation in comparison to no conversation.

Hancock et al. (2003) investigated the stopping decision of a group of cell phone distracted drivers using test track facilities where participants were instructed to perform a quick stop before reaching the stop line of an intersection upon the onset of a red light. They found that the non-response to a red light increased by $15 \%$ for drivers distracted by a cell phone visual-manual task consisting of looking down at a dial pad and confirming a number presented was the same as the number presented earlier. Using a driving simulator, Beede and Kass (2006) found that cell phone distracted drivers took one-third of a second longer before starting from a stop-sign indicating a slower response of distracted drivers. Consiglio et al. (2003) examined the braking performance of distracted drivers upon the activation of a red brake lamp set at a laboratory station and found that both hands-free and hand-held cellular phone conversation significantly increased the reaction time to braking events initiated by the lamp. Haque and Washington (2014b) examined the braking behavior of distracted drivers in response to a pedestrian entering a zebra crossing, and reported that drivers distracted either by handheld or hands-free phone conversation are associated with aggressive braking compared to non-distracted drivers, revealing perhaps an element of risk compensation. Strayer and Johnston (2001) examined the effects of cellular phone conversation on driving performances using a desktop simulator study, where participants performed a pursuit tracking task with a joystick and responded to flashing signals on a computer display. The study reported that drivers distracted by hand-held or hands-free conversation were two times 
less likely to detect simulated traffic signals and exhibited slower reactions to those signals that were detected.

In summary, using a cell phone while driving appears to influence many common driving behaviors including a deterioration of speed control, reduction in speed, failure to maintain appropriate headway, increase in lane position variation, reduction of peripheral eye scanning, decline in breaking performances, and impairment in the perception of relevant stimuli (e.g., Regan, Young, \& Lee, 2009). In particular, the slower reaction and impaired braking performances of distracted drivers might affect their safety at the onset of yellow lights at signalized intersection. However, there is little research on how distracted drivers perform at the onset of yellow lights.

\subsection{Driver Behavior at the Onset of Yellow Light}

Yellow light or phase changing period, the time interval when drivers need to decide on stopping or proceeding through an intersection, has been identified as a critical interval at signalized intersections (e.g., Elmitiny, Yan, Radwan, Russo, \& Nashar, 2010; Papaioannou, 2007). An improper decision at the onset of a yellow or amber light might cause red light running or abrupt stopping at intersections. It was estimated that red light running is associated with about 260,000 crashes on U.S. roads each year, of which about 750 are fatal (Retting, Ulmer, \& Williams, 1999). Earlier research on the driver's stop/go decisions at the onset of yellow lights has mainly been focused on developing a model to estimate the propensity of yellow running as a function of driving speed, distance from the stop line, and drivers' demographics like age and gender. Papaioannou (2007) reported that drivers who have an approach speed higher than the posted speed limit are more likely to be caught in a dilemma zone that might force them to make unsafe decisions at the onset of yellow light. Elmitiny et al. (2010) analyzed video-based data of a high-speed signalized intersection and found that red light violations and drivers' stop/go decisions during the yellow light are significantly associated with their distance from the stop line, operating speed, and position in the traffic flow. Using a driving simulator, Caird et al. (2007) investigated younger and older drivers' behavior at the onset of yellow light at signalized intersections and found that all drivers are less likely to run through the yellow light when their time to stop line is higher. Ohlhauser et al. (2011) investigated the effects of distraction at the onset of yellow light across various age cohorts. They reported that compared to middle-aged drivers, novice drivers are more likely to proceed through the intersection when distracted by a handheld phone conversation.

\subsection{Research Objective}

Drivers' stop/go decisions have previously been modeled in relation to driver demographics such as age and gender, and traffic parameters such as speed and distance from the stop line. It is of interest to examine whether distraction will impact a driver's response to the onset of a yellow interval while approaching a signalized intersection. In particular, it is worthy to investigate how a cell phone distraction interacts with age, gender differences and different traffic parameters in relation to the stop/go decisions of drivers at the onset of yellow light. While it might be difficult to expose distracted drivers at yellow light encounters in real world settings repeatedly, a driving simulator could be fully utilized to include such scenarios. The objective of this study is to examine the decisions of distracted drivers at the onset of yellow lights by exposing a group of distracted drivers on a series of signalized intersections using the National Advanced Driving Simulator at the University of Iowa. 


\section{Experiment Details}

\subsection{Participants}

The data analyzed in this study were gathered from two separate studies conducted at the University of Iowa: a wireless urban arterial study and a novice driver study (Mazzae, Goodman, Garrott, \& Ranney, 2005; Ranney et al., 2005). The wireless urban arterial study on three adult groups was conducted in the summer of 2004, and the novice driver study on 16-17 years old male drivers was conducted between May and October of 2006. Details of recruitment, screening, and compensation are available in Marshall et al. (2010).

In total, there were 69 drivers including 49 adults and 20 novice drivers. A valid driver's license was the main criterion for adult drivers and at least 4 weeks but no more than 8 weeks of licensure was the criterion for novice drivers. While the 20 male novice drivers of 16-17 years old formed a separate group, the 49 adult participants were divided into the following three age groups: younger (18-25 years old including 9 male and 9 female), middle-aged (30-45 years old including 9 male and 8 female), and older (50-60 years old including 8 male and 6 female). The average driving experiences for novice, younger, middle, and older drivers were $0.1,5.5,19.8$, and 36.7 years, respectively.

\subsection{Driving Simulator}

Both studies were conducted in the high fidelity NADS-1 simulator located at the University of Iowa. It is known to be the most advanced of its kind, consisting of an entire car housed inside a 24-feet dome providing 360-degree high resolution field-of-view to drivers. Road images and interactive traffic are updated at $60 \mathrm{~Hz}$ on eight LCD projectors to provide a photorealistic virtual environment. It is comprised of a 13 degree-of-freedom motion base to accurately reproduce motion cues for sustained acceleration and braking maneuvers, movement across multiple lanes of traffic, and interaction with varying road surfaces. Driving performance data such as lane position, speed, acceleration, and braking are recorded at rates up to $240 \mathrm{~Hz}$.

\subsection{Procedure}

Prior to the experiment, each participant, including the parent/guardian of novice drivers, completed an informed consent. After providing an overview of the study drives, participants were briefed about how to use the simulator and the cell phone apparatus. Before participating in the experimental drive, each participant performed a practice drive to be familiar with the driving simulator.

The traffic scenarios and distracting tasks were the same for both studies, and details are provided in Ranney et al. (2005) and Marshall et al. (2010). While the aim of Ranney et al. (2005) was to examine the effects of wireless phone use on driver performance for adults, the aim of Marshall et al. (2010) was to examine the effects of the same distracting task on novice drivers (16-17 years old). Although these original studies did not focus specifically on the risk of yellow light running, they used the same simulator protocol and contained detailed scenarios for traffic light change on a series of signalized intersections.

In summary, the driving route on the NADS-1 simulator contained three segments (approximately 15 minute each) with each segment containing both an urban and rural area. Five controlled intersections were included in each segment (or 15 total). The analyses in this paper focused on the urban areas where only two signalized intersections existed per segment (or six total). Programs were scripted so that the traffic light turned from green to yellow 
when the host vehicle was at 3.0 seconds from the stop line in one intersection and at 3.75 seconds from the stop line in another. The yellow phase was also 3.0 and 3.75 seconds respectively to generate a "dilemma zone" situation. The time to activate the yellow lights was counterbalanced across all segments. The road consisted of four lanes (12 feet each) with two lanes in each direction. There was ambient traffic traveling in the same direction as the participant (northbound) at level of service A (free flowing) and there was no traffic in the opposite direction. The posted speed limit on the approach was $45 \mathrm{mph}$. At the 4-way signalized intersection of interest, there was no other traffic present during the dilemma zone.

Among the three driving segments, one segment was used as a baseline condition, i.e., without any distraction task and the other two segments involved participants talking with the experimenter using a handheld phone interface. That is, each participant drove through two intersections without any conversation task and four intersections with a phone conversation task. This included placing a call and answering a call. Driving events such as change of traffic light from green to yellow occurred while participants were in the conversation phase of the call. The order of the driving segments for baseline and phone conversation tasks was counterbalanced across participants. It should be noted that proceeding through a yellow light does not necessarily comprise a surprise event given that there is no possibility of the driver colliding into another vehicle. Hence, it is reasonable to have repeated events per participants. Further, running a yellow light does not necessarily indicate an undesirable outcome nor is it considered a moving violation in the US.

A modified version of the Baddeley working memory span task was used (Baddeley, Logie, Nimmo-Smith, \& Brereton, 1985; Donmez, Boyle, \& Lee, 2006) to engage participants in conversation while driving. The working memory span task requires simultaneous storage and processing of information, and thus distracts the drivers by increasing the cognitive loads. In this task, participants were required to listen to sentences and determine whether they made sense. The experiment was designed to ensure that the conversation lasted for the duration of the driving events.

\subsection{Data Collection}

The driver's decision to proceed through or stop at the onset of yellow light was extracted as a binary outcome from simulator data and included as the dependent variable. Explanatory variables included driver's age group, gender, phone condition and speed at the onset of yellow. Phone condition variable had two categories: baseline and handheld conversation.

Traffic lights in the driving simulator were programmed using a time to stop line (TSL) variable, which represents the time required for a participant's vehicle to reach the stop line based on their speed and distance from the stop line at the onset of yellow light. In particular, the traffic lights were programmed to change from green to yellow when the participants were 3 or 3.75 seconds from the stop line. To account for any variability, TSL at the onset of yellow light was calculated from the dataset and included as a continuous variable for analysis. The minimum and maximum TSL were 2.47 and 3.80 seconds respectively. Mean TSL was 3.27 seconds with a standard deviation of 0.37 .

In total, there were 414 observations for 69 drivers encountering a traffic light change in six urban intersections along three road segments. Driving performance data such as speed of the driven car and distance from the stop line at the onset of yellow in five intersections were not correctly captured by the driving simulator due to a data recording error. As a result, there were 409 total observations for 69 drivers representing an unbalanced panel data with minimum 2 and maximum 6 observations per driver. Summary statistics of the variables for the decision tree and subsequent logistic regression model are presented in table 1 . There were 119, 108, 102 and 80 encounters with the traffic light change at signalized intersections 
43

for novice, young, middle-aged and older drivers, respectively. Among them 136 encounters with traffic light change happened in baseline or no phone conversation driving, while the rest (273) encounters happened in handheld phone conversation driving. The mean driving speed at the onset of yellow light was $42.5 \mathrm{mph}$ with a standard deviation of 5.2. Among the total 409 observations at signalized intersections, drivers decided to proceed through a yellow light in 153 encounters with the traffic light change.

\section{Statistical Modeling}

A repeated measures logistic regression model in the form of Generalized Estimation Equations (GEE) was applied to account for multiple observations across individuals. GEEs are an extension of generalized linear models to analyze correlated data, where the correlation is the result of repeated observations of the same driver at multiple points in time. GEEs are quite flexible in that they can accommodate non-normal variables and non-linear relationships well. In GEEs, the marginal expectation of the dependent variable is specified as a known linear function of covariates, assuming that the variance is a known function of the mean. In addition, GEEs specify a 'working' correlation matrix for the observations of each driver (Liang \& Zeger, 1986).

Suppose, the binary outcome of driver's decision to proceed through or stop at the onset of yellow light for driver $i$ at intersection $j$ is $Y_{i j}\left(i=1,2, \ldots, k ; j=1,2, \ldots, n_{i}\right)$. In the logistic regression model, the marginal expectation of, $E\left(Y_{i j}\right)=\mu_{i j}$, satisfies $\log i t\left(\mu_{i j}\right)=\mathbf{X}_{i j}^{\prime} \boldsymbol{\beta}$, where $\boldsymbol{X}_{i j}^{\prime}=\left(X_{i j}, \ldots, X_{i j p}\right)^{\prime}$ denote a $P \times 1$ vector of explanatory variables, and $\boldsymbol{\beta}$ is a vector of estimable regression parameters. Then the probability of yellow light running of driver $i$ could be expressed as:

$$
\operatorname{Pr}\left(Y_{i j}=1\right)=\frac{\exp \left(\mathbf{X}_{i j}^{\prime} \boldsymbol{\beta}\right)}{1+\exp \left(\mathbf{X}_{i j}^{\prime} \boldsymbol{\beta}\right)}
$$

Suppose, $V_{i}$ is an estimator of the covariance matrix of $Y_{\mathrm{i}}$, then $\boldsymbol{\beta}$ can be estimated by solving the GEEs as follows:

$$
S(\beta)=\sum_{i=1}^{k}\left(\frac{d \mu_{i}^{\prime}}{d \beta}\right) V_{i}^{-1}\left(Y_{i}-\mu_{i}(\beta)\right)=0
$$

The estimator of the covariance matrix is specified as $V_{i}=\phi A_{i}^{1 / 2} R_{i}(\rho) A_{i}^{1 / 2}$, where $A_{i}$ is a $n_{i} \times$ $n_{i}$ diagonal matrix with $v\left(\mu_{i j}\right)$ as the $j^{\text {th }}$ diagonal element. $V_{i}$ can be different from one subject to another, but it is common to specify the same form of $V_{i}$ for all subjects. $R_{i}(\rho)$ is a $n_{i} \times n_{i}$ working correlation matrix that is fully specified by the vector parameters $\rho$. An exchangeable working correlation that makes constant correlations between any two observations within a subject is specified as:

$$
R_{6 x 6}=\left[\begin{array}{llllll}
1 & \rho & \rho & \rho & \rho & \rho \\
\rho & 1 & \rho & \rho & \rho & \rho \\
\rho & \rho & 1 & \rho & \rho & \rho \\
\rho & \rho & \rho & 1 & \rho & \rho \\
\rho & \rho & \rho & \rho & 1 & \rho \\
\rho & \rho & \rho & \rho & \rho & 1
\end{array}\right]
$$


Detailed expressions for estimating $\rho$ 's are available in Liang and Zeger (1986). In addition, robust variance estimates have been applied because they are agnostic about the nature of the interdependence in the data (Zorn, 2006). That is, the robust variance estimates do not depend on whether the conditional correlation among observations is positive or negative and thus provides better estimates of the parameters of interest.

Selecting the best subset of explanatory variables that includes main effects, nonlinearities, and interactions effects is often challenging, especially when little prior experience guides a specification. This is because traditional approaches require the analyst to specify a priori second- and higher-order interactions, non-additive behaviour, and nonlinearities of the main effect variables prior to running stepwise or best subsets procedures. In many explanatory research settings, the number of the potential combinations of variables and their higher-order interactions grow geometrically with the number of ordinal-scale variable and exponentially with nominal-scale variables. Therefore, it is difficult to decide which interactions to test and which to omit.

To circumvent this problem, an iterative combination of decision tree and logistic regression analyses was applied in this study. Decision or classification and regression trees are nonparametric methods; they are exploratory in nature and are helpful for uncovering possible relationships by selecting an appropriate explanatory variable in each stage to produce a maximum reduction in variability of the dependent variable; however, decision trees suffer from type I errors and lack the ability to make inferences. Despite these limitations, the results of a decision tree can be utilized to provide a priori knowledge for the logistic regression model by identifying interactions indicated by the tree branches. Therefore, this combined modeling technique allows exploiting the best of both qualities: possible higher order interactions by decision trees and inferences by the logistic regression model (Washington, 2000). The general procedures for including higher order interactions can be summarized in the following steps:

1. Estimate a decision tree to reveal patterns and relationships of the sampled data.

2. Convert the interactions identified by the decision tree into indicator variables.

3. Estimate a logistic regression model with both main effect variables and interaction variables.

4. Retain significant interactive variables, prune the tree and re-identify possible interactions

5. Iterate steps 3-4 until a theoretically and practically appealing justifiable specification of the logistic regression model is obtained.

In summary, the combined approach provides researchers a priori knowledge to select interaction terms and is helpful for conducting exploratory research and developing statistically and theoretically defensible model specifications.

In order to interpret the effect of coefficient estimates, the exponents of the parameter estimates (i.e. $\exp (\beta))$ were calculated to obtain the odds ratios (ORs) which provide the magnitude of the association between the factor of interest and the probability of running a yellow light. ORs greater than 1 indicate increased probability whereas ORs less than 1 indicate decreased probability. For categorical variables, $\exp \left(\beta_{a}-\beta_{b}\right)$ is used to represent the odds ratio between two categories. All parameter estimates were mainly assessed at $5 \%$ significance level, and ORs are reported with the corresponding 95\% confidence intervals (CI). 


\section{Results}

A decision tree was constructed from available data to classify discrete outcomes of driver's stop/go decision at the onset of yellow indications at signalized intersections. Variables like driver's age group, gender, distance to stop line, speed, and time to stop line (TSL) were offered as possible explanatory variables in the decision tree. Figure 1 illustrates the decision tree diagram for the stop/go decision model. Nodes were determined selecting the option that offered the highest information gain. The decision tree was constructed using 10-fold stratified cross-validation, which divided the data into ten unique partitions, which were each used in turn to test the decision tree. Thus, on each cycle nine-tenths of the data was being used to train the decision tree. The decision tree correctly classified $60 \%$ of instances, using 15 leaves for a total tree size of 27 nodes. Driver's age represents the highest information gain and is therefore at the top of the tree (as shown in Figure 1). Higher order interactions have been found for young drivers with gender, speed at yellow and TSL, middle-aged drivers with gender, phone condition, TSL and distance from the stop line at yellow, and older drivers with phone condition and TSL.

The decision tree classified drivers' stop/go decisions through segmenting the dataset into 15 smaller and more homogenous groups. The group statistics that indicate the classification rules for the stop/go decision are presented in the parentheses at the bottom of each branch of the tree in figure 1 . The numbers in parenthesis below the leaves indicate how many instances reached the leaf (first number), and of those, how many were not classified as part of the branch (second number). For example, the statistic of branch 1 suggests that 119 novice drivers reached the leaf, and of these, $71(71 / 119=59.7 \%)$ would stop at the onset of yellow light at signalized intersections. Similarly, branch 2 implies that about $76.3 \%(29 / 38)$ of young females would stop and $23.7 \%$ (9/38) of them would proceed through an intersection when the speed at the onset of yellow is less than or equal to $45.5 \mathrm{mph}$. Branch 12 suggests that about $65 \%$ of distracted older drivers would proceed through the intersection when the time to stop line at the onset of yellow is less than or equal to 3.7 seconds.

Using the decision tree, 15 interaction variables were created and shown in third brackets at the bottom of each tree branch. For example, interaction variable 2 refers to a young female driver whose speed at the onset of yellow is less than or equal to $45.5 \mathrm{mph}$. Similarly, interaction variable 8 refers to a situation where a middle-aged female driver driving in the handheld phone condition and the vehicle's distance from the stop line at the onset of yellow is greater than $162.8 \mathrm{ft}$ and the TSL is less than or equal to 3.72 seconds. Interaction variable 12 refers to a distracted older driver with the TSL at the onset of yellow is less than or equal to 3.7 seconds. These higher order interaction variables coupled with main effect variables and all possible second-order interaction variables were fitted into the repeated measures logistic regression model described previously. Significant variables were retained to derive the parsimonious model.

The significant variables estimated from the logistic regression model along with the probabilities of drivers' decision for proceeding through the intersection at the onset of a yellow light are reported in table 2. The best-fitted model yielded a Wald chi-square statistic of 31.1 with $7 \mathrm{df}$, which is well above the critical value at $5 \%$ significance level, implying that the model has sufficient explanatory power. Moreover, a value of 0.39 for the exchangeable correlation parameter, $\rho$ indicates that there is a significant correlation among observations of each driver and further ensures the appropriateness of the repeated measures logistic regression model for this dataset.

The parsimonious model included three main effect variables: speed at the onset of yellow light, phone condition, and driver's gender, and four higher order interaction variables: interaction variable $2,8,10$, and 13 from the decision tree. The parameter 
estimates are explained in this section in terms of the odds ratio. That is, each variable's influence on the odds of running a yellow signal is explained while controlling for other effects in the model. However, it is important to recognize that the likelihood of proceeding through a yellow light is a function of many factors, which are described in more detail in the Discussion.

Speed at the onset of yellow was a significant predictor at 5\% significance level in explaining driver's decision at the onset of a yellow light at intersections. Results indicate that drivers are more likely to proceed through the intersection with an increased speed. An estimate of odds ratio $[\operatorname{Exp}(\beta)]$ indicates that a $1 \mathrm{mph}$ increase in speed is associated with about $4 \%$ increase in driver's probability to proceed at the onset of yellow.

The phone condition variable suggests that overall, the odds of proceeding through a yellow light decreased by $33 \%$ when participants were talking with a handheld phone. It should be noted that the odds ratio estimate does not take into account the effects of interaction variables related to phone condition such as interaction variable 8 and interaction variable 13. Since this study has included a full version of interaction variables, the varied effects of cell phone distraction would be identifiable while interpreting interactions of cell phone distraction with other variables such as driver's age, gender and other situational parameters.

Driver's gender was significant at the 5\% level and indicated that female drivers are more likely to run through the yellow light compared to males. The corresponding odds ratio suggests that female drivers are 2.62 times more likely to proceed through a yellow light compared to males.

The driver's age factor was grouped into four classes: novice (16-17 years), young (18-25 years), middle-aged (30-45 years) and older (50-60 years). Middle-aged group was used as a reference category for the modeling purpose. None of the main effect variables of age group was significant in the repeated measures logistic regression model. However, the effects of age were evident in the model through four higher order interaction variables derived from the decision tree. While young and older drivers have a higher order interaction each, middle-aged drivers have two higher order interactions in the model.

A higher order interaction variable of young drivers shows that young female driver group has a significant association in driver's stop/go decision model with respect to speed thresholds. Interaction variable 2 indicates that a young female driver, when drives slower than $45.5 \mathrm{mph}$, is less likely to proceed through an intersection with the corresponding odds about $70 \%$ lower.

Middle-aged female drivers have significant associations in the logistic regression model with respect to time to stop line (TSL), distance from the stop line at the yellow and phone condition. Interaction variable 8 suggests that a middle-aged female driver driving in cell phone distracted condition is about $71 \%$ less likely to proceed through a yellow light when the vehicle's distance at the onset of yellow light is greater than $162.8 \mathrm{ft}$ and the TSL is less than or equal to 3.72 seconds. In contrast, non-distracted driving of a middle-aged female driver appears to have different TSL thresholds in the driver's stop/go logistic regression model. Interaction variable 10 indicates that a middle-age female driver driving without a cell phone distraction is about $83 \%$ less likely to run the yellow light if their distance at the onset of yellow is greater than $162.8 \mathrm{ft}$ and the TSL is in between 2.95 and 3.72 seconds.

Older driver group has a significant association with TSL thresholds in distracted driving condition. Interaction variable 13 suggests that distracted older drivers are about 6.1 times more likely to run the yellow light if their TSL at the onset of yellow is greater than 3.7 seconds.

To examine the differential risk and behavior arising from distraction and approach speed, a complex model accounting for the interaction terms can be used. To illustrate, we 
might be interested in comparing the probabilities of running a yellow signal of distracted older male drivers at approach speeds of 30 and $40 \mathrm{mph}$. We can calculate the relevant probabilities for each case using logistic regression equation 1. Probabilities of yellow light running of a discrete group of individuals at different approach speed values could be computed by using corresponding $\mathbf{X}$ values and parameter estimates, while holding other variables at their reference category values. Relevant parameter estimates for distracted older male drivers are constant, speed at yellow, handheld phone and Interaction variable 13. Taking the corresponding parameter estimates from table 2, the predicted probabilities for this group of driver at approach speed $=30 \mathrm{mph}$ and approach speed $=40 \mathrm{mph}$ are calculated as follows:

$$
\begin{aligned}
& P_{\text {Distracted Older Male Speed }=30}=\frac{\operatorname{EXP}[-2.12+0.04(30)-0.406(1)+1.813]}{1+\operatorname{EXP}[-2.12+0.04(30)-0.406(1)+1.813]}=0.62 \\
& P_{\text {Distracted Older Male Speed }=40}=\frac{\operatorname{EXP}[-2.12+0.04(40)-0.406(1)+1.813]}{1+E X P[-2.12+0.04(40)-0.406(1)+1.813]}=0.71
\end{aligned}
$$

Using this approach for a variety of driver groups, figure 2 presents the model predicted probabilities of running a yellow light as a function of approach speed. Since observed approach speeds of drivers ranged between 25 and $55 \mathrm{mph}$, the yellow light running probabilities are plotted for that speed range. Findings from this plot have been elaborately discussed in the next section.

\section{Discussion}

On average and without the effects of interaction variables, participants in this study are more likely to stop at the onset of a yellow light when engaged in a cell phone conversation (odds ratio 0.666). This effect might be explained by risk compensation-where the sampled drivers' compensated for the increased risk induced by distraction by reducing their willingness to run the yellow light. During a conversation, a driver may have reduced mental capacity to attend to the driving task, which could be perceived as a reduction in the ability to respond quickly to a hazardous situation. It is also possible that the increased workload of talking on the phone results in resources allocated away from other tasks, one of them being the assessment of the risks of running the yellow light. While this effect may appear to be risk compensation, it could simply be the result of cognitive resource reallocation. From this study, it is not possible to know whether risk compensation or cognitive resource allocation has resulted in reduced yellow light running. There are a number of studies that associate crash risks with engagement in cell phones while driving (e.g., Neyens \& Boyle, 2007; Redelmeier \& Tibshirani, 1997). Hence, if risk compensation is occurring, it is likely to be insufficient to offset increased crash risk caused by distraction.

Risk compensation of distracted drivers has been repeatedly noted in the literature as a possible explanation for observed speed reductions (e.g., Haigney, Taylor, \& Westerman, 2000; Törnros \& Bolling, 2006). It has generally been argued that drivers tend to compensate for the increase workload of talking on their cell phone whilst driving by selecting a lower driving speed. To test whether any speed reduction was evident in this dataset, drivers' approach speeds to a signalized intersection were tested and compared across phone conditions using the repeated measures ANOVA in the form of a Linear Mixed Model as used by Haque and Washington (2013). As shown in table 3, the difference in driving speed at the onset of yellow-measured at the instance when the traffic light changed from green to yellow-was statistically significant $\left(F_{1,339.84}=5.493, p\right.$-value $\left.=0.020\right)$ across phone conditions. The mean speed at yellow in baseline driving was $43.2 \mathrm{mph}$, while the 
corresponding speed in cell phone conversation condition was 42.2. On average, drivers tended to drive $1 \mathrm{mph}$ slower while driving distracted.

Overall, the results suggest that drivers tend to compensate for their increased attention load of cell phone conversation by not only reducing the probability of yellow light running but also selecting a lower speed while approaching to a signalized intersection. In contrast, other research has shown that drivers engaged in a phone conversation are more likely to miss (Strayer \& Johnston, 2001) or react slower to critical signals (Consiglio et al., 2003) and changing stop lights (Hancock et al., 2003). Drivers' responses to cell phone distraction and changes in traffic lights appear to behave in complex ways, as is evident from many higher order interactions in the logistic regression model. A closer examination of driver behavior mainly comparing yellow running probabilities across different driver groups (e.g. young male, older female, etc.) is provided later in this section.

The model suggests that females are less likely to stop for a yellow signal. The average approach speed of female drivers at the onset of yellow light was $42.2 \mathrm{mph}$, while the corresponding speed for male drivers was $42.7 \mathrm{mph}$. The speed difference between males and females is not statistically significant $(p$-value $=0.313)$. This insignificant difference in speed combined with females being less likely to stop implies that females may be less responsive to traffic light changes when compared to males traveling at similar speeds.

The risk of running a yellow signal increases by a factor of $4 \%$ (odds ratio 1.04) for those who travel faster than the mean speed by $1 \mathrm{mph}$. Similar findings were also observed in other studies (e.g., Papaioannou, 2007) where drivers were more likely to proceed through an intersection when their approach speeds were higher.

The presence of high order interaction effects indicates some complexity in the relationships between driver's stop/go decision at the onset of yellow and operating speed, distance from the stop line, time to stop line (TSL), phone condition and driver demographics including age and gender. The effects of cell phone distraction on the speed selection of drivers approaching signalized intersections have further complicated these relationships. To examine the differential risk, probabilities of yellow light running across various driver groups are plotted in figure 2 .

As shown in figure 2, the probability of yellow light running across all age groups generally tends to increase with the approach speed at the onset of yellow light. For example, the probability of yellow light running for non-distracted male drivers at $25 \mathrm{mph}$ is $25 \%$, while at an approach speed of $55 \mathrm{mph}$ the corresponding probability is $42 \%$. Both males and females have a lower probability of yellow light running across the whole speed range when they are engaged in a cell phone conversation while driving. For instance, the probability of yellow running of female drivers at $45 \mathrm{mph}$ is about $10 \%$ lower in distracted condition compared to non-distracted driving.

Distracted female drivers, however, have a higher probability of yellow light running across the whole speed range compared to distracted male drivers. The probability of yellow running at $45 \mathrm{mph}$, for instance, for distracted female drivers is $56 \%$ while the corresponding probability for distracted males is only $33 \%$. Speed selection of female drivers also seems to be influenced by the cell phone conversation task. As reported in table 3 , the difference in driving speed across phone conditions was not statistically significant $\left(F_{1,227.76}=1.365\right.$, $p$ value $=0.245)$ for male drivers but it was significant $\left(F_{1,110.95}=7.366\right.$, $p$-value $\left.=0.008\right)$ for female drivers. While the driving speed of non-distracted female drivers at the onset of yellow was about $43.4 \mathrm{mph}$, the corresponding speed for distracted females was about 41.6 mph. In summary, the effects of distraction in terms of both yellow light running and speed reduction appear to be higher among female drivers than males.

Both young male and female drivers appear to reduce their yellow light running probabilities in the distracted driving condition across the whole speed range, with the 
corresponding probability on average about $10 \%$ lower in the distracted condition compared to normal driving. Interestingly the probability of yellow light running of young females in both distracted and non-distracted conditions increases significantly, when the approach speed exceeds the posted speed limit of $45 \mathrm{mph}$. At an approach speed of $45 \mathrm{mph}$, the probability of yellow light running of distracted female drivers is $28 \%$ while the corresponding probability at the approach speed of $50 \mathrm{mph}$ is $61 \%$. Drivers travelling faster than the speed limits or the speed values used for determining the traffic signal time settings are likely to be caught in a dilemma zone, where they can neither cross the intersection without red light running nor stop without hard braking (Papaioannou, 2007). The effect of the dilemma zone appears to have a greater influence on young female drivers as their probability of yellow running is significantly increased when their approach speed exceeds the speed limit.

Like young males, middle-age male drivers have a similar trend of yellow light running, with a similar reduction in yellow running probabilities in distracted condition. However, the difference of yellow running probabilities in distracted and baseline driving of middle-aged female drivers is marginal across the whole speed range. On average, middleaged female drivers have a lower probability of yellow running compared to middle-aged males.

In contrast, older drivers appear to have an opposite pattern in yellow light running risk. Both older males and females have a higher probability of yellow light running in distracted driving condition compared to the baseline across the whole speed range. For instance, the probability of yellow light running at the approach speed of $45 \mathrm{mph}$ for a nondistracted older male driver is about $42 \%$ while the corresponding probability for a distracted older male is as high as $75 \%$. On average, the probability of yellow light running of older males is about $33 \%$ higher in the distracted driving condition and the difference is constant across the whole speed range. Older female drivers exhibit the same effect, and it marginally decrease with increasing speed; that is the probability difference decreases with approach speed. At low approach speeds (say $30 \mathrm{mph}$ ), the probability of yellow running of older females is about $30 \%$ higher in distracted condition compared to baseline driving, while the corresponding probability difference at an approach speed $55 \mathrm{mph}$ is about $18 \%$. Previous research (e.g., Knoblauch et al., 1995) has documented that average slower response times of older drivers might impair their actions at the onset of yellow light. This effect might be exacerbated by a cell phone distraction as evident in the yellow light probabilities of distracted older drivers. Apart from this, speed selection of older drivers along an approach of a signalized intersection also tends to be significantly $\left(F_{1,66.00}=6.131, p\right.$-value $\left.=0.016\right)$ affected by the cell phone conversation task (see table 3). Approach speed of older driver in baseline driving condition was about $43.1 \mathrm{mph}$ while the corresponding speed in distracted condition was about $40.6 \mathrm{mph}$.

Compared with older male drivers, probabilities of yellow running across the entire speed range are higher among older females both in distracted and non-distracted driving conditions. For instance, the probability of yellow running at $45 \mathrm{mph}$ for distracted older males is $75 \%$, while the corresponding probability for older females is as high as $89 \%$. In addition, the cell phone distraction appears to have a significant effect on the speed selection of older females $\left(F_{1,25.69}=18.312\right.$, $p$-value $\left.<0.001\right)$ but not among older males $\left(F_{1,39.00}=\right.$ 0.833 , $p$-value $=0.367$ ). Average approach speeds along an approach of a signalized intersection for older females in distracted and baseline driving were 44.9 and $40.5 \mathrm{mph}$, respectively. That is the speed reduction was about $4.4 \mathrm{mph}$ due to the cell phone conversation task. In summary, the influence of a cell phone conversation on driving performance of older female driver group along an approach to signalized intersection appears to be the most among all driver groups. 


\section{Conclusions}

This study applied a combination of data mining and classical statistical modeling techniques to examine distracted drivers' stop/go decisions at the onset of yellow lights at signalized intersections. Data were obtained from a group of drivers on a motion-based driving simulator (NADS-1). The methodology combined logistic regression and decision trees in order to leverage the strengths of both approaches - an ability to test significance of observed effects with an ability to heuristically explore the data for previously unknown relationships. The combined methodology provided useful insights regarding interaction effects that significantly influenced drivers' decisions to proceed through a yellow light while distracted by a cell phone conversation.

Overall, the model suggests that driver responses to distraction and running a yellow signal vary considerably across gender and age. Female drivers have a higher probability of running through a yellow light in general. Risk compensation, or complexity compensation, was observed in young and middle-aged driver groups. Distracted young and middle-aged drivers are predicted to compensate for the increased mental load and/or crash risk of distraction by running the yellow signal with lower probability. It is not clear from this study whether drivers were responding to increased perception of risk or simply an increased cognitive workload. However, yellow light running is associated with crash risk, and this study showed that distracted young and middle-aged drivers reveal a reduction in their willingness to accept as much risk as when not distracted. In contrast, older drivers appear to behave quite differently in the presence of distraction in this scenario and predicted by this model. Overall, older drivers have a higher probability of yellow light running while distracted by a cell phone conversation. In particular, the performance of older females appears to be the most affected by the cell phone distraction.

Speed selection of drivers on an approach to a signalized intersection also appears to be influenced by the cell phone conversation task. In general, drivers tended to select lower speeds when they were engaged in cell phone conversations. In particular, female drivers tended to reduce their driving speeds while distracted by the cell phone conversations, unlike males. Distracted older drivers tended to select slower approach speeds to traffic signals. Hence, the effects of distraction on speed reduction as well as yellow running appear to be higher for these two groups of drivers.

This study was based on a reanalysis of existing data and as such, there were some limitations given that the original study goals were not aligned with the aims of this paper. The analysis was based on two different studies (albeit the same protocol), there may be differences between novice drivers and the other three age groups that may not be related to age, but rather some subtle differences in each study goal (one was specifically on use of wireless devices, while the other was on novice driver risk propensity). Furthermore, the novice drivers consisted of only males and as such, any differences in gender for novice drivers could not be detected. It is also recognized that drivers may exhibit different responses between initial and final exposure to a yellow light given learning effects, which may also bias outcomes. Hence, additional simulator studies would be useful to capture the changes in perception and workload, as well as to identify and test ways to mitigate the effects of driver distraction at intersections.

\section{Acknowledgments}

The authors would like to acknowledge the National Science Foundation for funding the novice driver study, and the US DOT - National Highway Traffic Safety Administration for funding the wireless urban arterial study. We also acknowledge Ginger Watson, PhD, 
original Principal Investigator at NADS, who contributed significantly to the study design and early stages, Elizabeth Mazzae and Flaura Winston, the Principal Investigators, and Omar Ahmad who provided the data.

\section{References}

Baddeley, A., Logie, R., Nimmo-Smith, I., \& Brereton, N. (1985). Components of fluent reading. Journal of Memory and Language, 24(1), 119-131. doi: 10.1016/0749$596 x(85) 90019-1$

Beede, K. E., \& Kass, S. J. (2006). Engrossed in conversation: The impact of cell phones on simulated driving performance. Accident Analysis \& Prevention, 38(2), 415-421. doi: 10.1016/j.aap.2005.10.015

Burns, P. C., Parkes, A., Burton, S., Smith, R. K., \& Burch, D. (2002). How dangerous is driving with a mobile phone? Benchmarking the impairment to alcohol. Crowthorne, UK: TRL Limited.

Caird, J. K., Chisholm, S. L., Edwards, C. J., \& Creaser, J. I. (2007). The effect of yellow light onset time on older and younger drivers' perception response time (PRT) and intersection behavior. Transportation Research Part F: Traffic Psychology and Behaviour, 10(5), 383-396. doi: 10.1016/j.trf.2007.03.002

Consiglio, W., Driscoll, P., Witte, M., \& Berg, W. P. (2003). Effect of cellular telephone conversations and other potential interference on reaction time in a braking response. Accident Analysis \& Prevention, 35(4), 495-500. doi: 10.1016/s0001-4575(02)000271

Donmez, B., Boyle, L. N., \& Lee, J. (2006). The impact of distraction mitigation strategies on driving performance. Human Factors, 48(4), 785-804.

Dula, C. S., Martin, B. A., Fox, R. T., \& Leonard, R. L. (2011). Differing types of cellular phone conversations and dangerous driving. Accident Analysis \& Prevention, 43(1), 187-193. doi: 10.1016/j.aap.2010.08.008

Elmitiny, N., Yan, X., Radwan, E., Russo, C., \& Nashar, D. (2010). Classification analysis of driver's stop/go decision and red-light running violation. Accident Analysis \& Prevention, 42(1), 101-111. doi: 10.1016/j.aap.2009.07.007

Haigney, D. E., Taylor, R. G., \& Westerman, S. J. (2000). Concurrent mobile (cellular) phone use and driving performance: task demand characteristics and compensatory processes. Transportation Research Part F: Traffic Psychology and Behaviour, 3(3), 113-121. doi: http://dx.doi.org/10.1016/S1369-8478(00)00020-6

Hancock, P. A., Lesch, M., \& Simmons, L. (2003). The distraction effects of phone use during a crucial driving maneuver. Accident Analysis \& Prevention, 35(4), 501-514. doi: 10.1016/s0001-4575(02)00028-3

Haque, M. M., \& Washington, S. (2013). Effects of mobile phone distraction on drivers' reaction times. Journal of Australasian College of Road Safety (ACRS), 24(3), 20-29.

Haque, M. M., \& Washington, S. (2014a). A parametric duration model of the reaction times of drivers distracted by mobile phone conversations. Accident Analysis \& Prevention, 62, 42-53. doi: http://dx.doi.org/10.1016/j.aap.2013.09.010

Haque, M. M., \& Washington, S. (2014b). The impact of mobile phone distraction on the braking behaviour of young drivers: A hazard-based duration model. Transportation Research Part C: Emerging Technologies(0). doi: http://dx.doi.org/10.1016/j.trc.2014.07.011

Ironside, R. (2011, November 11, 2011). Queensland drivers fined $\$ 25,000$ a day for using mobile phones behind the wheel, The Courier-Mail. Retrieved from 
http://www.couriermail.com.au/news/queensland/queensland-drivers-finded-25000-aday-for-using-mobile-phones-behind-the-wheel/story-e6freoof-1226192370566

Knoblauch, R., Nitzbug, M., Reinfurt, D., Council, F., Zegeer, C., \& Popkin, C. (1995). Traffic operations control for older drivers (Report No. FHWA-RD-94-119). Washington DC: Federal Highway Administration.

Lam, L. T. (2002). Distractions and the risk of car crash injury: The effect of drivers' age. Journal of Safety Research, 33(3), 411-419. doi: 10.1016/s0022-4375(02)00034-8

Liang, K.-Y., \& Zeger, S. L. (1986). Longitudinal Data Analysis Using Generalized Linear Models. Biometrika, 73(1), 13-22.

Marshall, D., Dow, B., \& Brown, T. (2010). Validation of the national advanced driving simulator for the study of young driver risk for the center for child injury prevention science I/UCRC. Iowa City: IA: National Advanced Driving Simulator.

Mazzae, E., Goodman, M., Garrott, W. R., \& Ranney, T. A. (2005). NHTSA's Resaerch Program on Wireless Phone Driver Interface Effects: U.S. Department of Transportation.

Neyens, D. M., \& Boyle, L. N. (2007). The effect of distractions on the crash types of teenage drivers. Accident Analysis \& Prevention, 39(1), 206-212. doi: http://dx.doi.org/10.1016/j.aap.2006.07.004

NHTSA. (2014). Traffic Safety Facts: Distracted Driving 2012. Washington DC, USA: National Highway Traffic Safety Administration.

Ohlhauser, A. D., Boyle, L. N., Marshall, D., \& Ahmad, O. (2011). Drivers' behavior through a yellow light: Effects of distraction and age. Paper presented at the Human Factors and Ergonomics Society 55th Annual Meeting - 2011.

Papaioannou, P. (2007). Driver behaviour, dilemma zone and safety effects at urban signalised intersections in Greece. Accident Analysis \& Prevention, 39(1), 147-158. doi: 10.1016/j.aap.2006.06.014

Rakauskas, M. E., Gugerty, L. J., \& Ward, N. J. (2004). Effects of naturalistic cell phone conversations on driving performance. Journal of Safety Research, 35(4), 453-464. doi: $10.1016 /$ j.jsr.2004.06.003

Ranney, T. A., Watson, G., Mazzae, E. N., Papelis, Y. E., Ahmad, O., \& Wightman, J. R. (2005). Examination of the Distraction Effects of Wireless Phone Interfaces Using the National Advanced Driving Simulator - Final Report on a Freeway Study: U.S. Department of Transportation.

Redelmeier, D. A., \& Tibshirani, R. J. (1997). The association between cellular telephone calls and motor vechicle collisions. New England Journal of Medicine, 336(7), 453458.

Regan, M. A., Young, K., \& Lee, J. (Eds.). (2009). Driver Distraction: Theory, Effects, and Mitigation. New York: CRC press.

Retting, R. A., Ulmer, R. G., \& Williams, A. F. (1999). Prevalence and characteristics of red light running crashes in the United States. Accident Analysis \& Prevention, 31(6), 687-694. doi: 10.1016/s0001-4575(99)00029-9

Strayer, D. L., \& Johnston, W. A. (2001). Driven to distraction: Dual-task studies of simulated driving and conversing on a cellular telephone. Psychological Science, 12(6), 462-466.

Törnros, J., \& Bolling, A. (2006). Mobile phone use - effects of conversation on mental workload and driving speed in rural and urban environments. Transportation Research Part F: Traffic Psychology and Behaviour, 9(4), 298-306. doi: 10.1016/j.trf.2006.01.008

Washington, S. (2000). Iteratively specified tree-based regression: theory and trip generation example. ASCE Journal of Transportation Engineering, 126(6), 482-491. 
1 WHO. (2011). Mobile phone use: A growing problem of driver distraction. Geneva: World $2 \quad$ Health Organization.

3 Zorn, C. (2006). Comparing GEE and Robust Standard Errors for Conditionally Dependent 4 Data. Political Research Quarterly, 59(3), 329-341.

5

6

7

8 
TABLE 1 Summary statistics of variables included in the model

2

\begin{tabular}{llll}
\hline Variables & $\begin{array}{l}\text { Count on encounters } \\
\text { with traffic light change }\end{array}$ & Mean & St. Dev. \\
\hline Driver Demographics & & & - \\
\hline Age & 119 & - & - \\
Novice & 108 & - & - \\
Young & 102 & - & - \\
Middle* & 80 & - & - \\
Older & & - & - \\
Gender & 275 & - & - \\
Male* & 134 & & - \\
Female & & & - \\
\hline Experiment Variables & & & \\
\hline Phone Condition & 136 & 42.52 & 5.17 \\
Baseline* & 273 & 3.27 & 0.37 \\
Handheld & - & 204.22 & 33.76 \\
Speed at yellow (mph) & - & - & - \\
Time-to-stop line (seconds) & - & 153 & - \\
Distance at yellow (ft) & & & - \\
Drivers' decision to proceed through a yellow light & 153 & & \\
\hline
\end{tabular}

3

*Reference category for categorical independent variables 
TABLE 2 Repeated measure logistic regression model predicting the likelihood of proceeding through a yellow light

\begin{tabular}{|c|c|c|c|c|c|c|c|}
\hline \multirow{2}{*}{ Explanatory Variables } & \multicolumn{2}{|c|}{ Parameter Estimate } & \multirow{2}{*}{ z-statistic } & \multirow{2}{*}{$p$-value } & \multirow{2}{*}{$\begin{array}{l}\text { Odds Ratio } \\
\text { (OR) }\end{array}$} & \multicolumn{2}{|c|}{$95 \%$ CI of OR } \\
\hline & Estimate & SE & & & & $2.5 \%$ & $97.5 \%$ \\
\hline Speed at yellow & 0.040 & 0.016 & 2.54 & 0.011 & 1.041 & 1.009 & 1.073 \\
\hline Handheld Phone & -0.406 & 0.143 & -2.84 & 0.004 & 0.666 & 0.504 & 0.882 \\
\hline Female & 0.963 & 0.420 & 2.29 & 0.022 & 2.620 & 1.150 & 5.968 \\
\hline Interaction variable 2 & -1.185 & 0.483 & -2.45 & 0.014 & 0.306 & 0.119 & 0.788 \\
\hline Interaction variable 8 & -1.264 & 0.656 & -1.93 & 0.054 & 0.283 & 0.078 & 1.023 \\
\hline Interaction variable 10 & -1.740 & 0.740 & -2.35 & 0.019 & 0.175 & 0.041 & 0.748 \\
\hline Interaction variable 13 & 1.813 & 0.847 & 2.14 & 0.032 & 6.131 & 1.167 & 32.219 \\
\hline Constant & -2.120 & 0.758 & -2.80 & 0.005 & & & \\
\hline
\end{tabular}

Number of observations 409

Number of groups

69

Wald Chi-sq

Degrees of freedom 7

$p$-value

$<0.001$

Exchangeable correlation $\quad 0.3860$

parameter, $\rho$

2: A young female driver whose speed at the onset of yellow is $\leq 45.5 \mathrm{mph}$

Interaction variable 8: A distracted middle aged female driver whose distance from the stop line at yellow is > $162.8 \mathrm{ft}$ and time to stop line is $\leq 3.72$ seconds

7 Interaction variable 10: A middle aged female driver without phone conversations whose distance from the stop

8 line at yellow is $>162.8 \mathrm{ft}$ and time to stop line is between 2.95 and 3.72 seconds

Interaction variable 13: A distracted older driver whose time to stop line at the onset of yellow is $>3.7$ seconds 
TABLE 3 Speed selection of different driver groups at the onset of yellow light

2

\begin{tabular}{|c|c|c|c|c|}
\hline \multirow[t]{2}{*}{ Speed at Yellow } & \multicolumn{2}{|c|}{ Phone Condition } & \multirow{2}{*}{$\begin{array}{c}\text { Significance by a Linear Mixed } \\
\text { Model }\end{array}$} & \multirow[t]{2}{*}{ Remark } \\
\hline & Baseline & Handheld & & \\
\hline All Drivers & 43.212 & 42.170 & $F_{1,339.84}=5.493, p=0.020$ & Significant \\
\hline \multicolumn{5}{|l|}{ Age } \\
\hline Novice & 42.914 & 42.261 & $F_{1,97.68}=0.558, p=0.457$ & Not significant \\
\hline Young & 44.017 & 43.285 & $F_{1,89.00}=0.948, p=0.333$ & Not significant \\
\hline Middle & 42.762 & 42.163 & $F_{1,84.00}=0.485, p=0.488$ & Not significant \\
\hline Older & 43.142 & 40.559 & $F_{1,66.00}=6.131, p=0.016$ & Significant \\
\hline \multicolumn{5}{|l|}{ Gender } \\
\hline Male & 43.123 & 42.471 & $F_{1,227.76}=1.365, p=0.245$ & Not significant \\
\hline Female & 43.398 & 41.558 & $F_{1,110.95}=7.366, p=0.008$ & Significant \\
\hline \multicolumn{5}{|l|}{ Age X Gender } \\
\hline Novice Male & 42.914 & 42.261 & $F_{1,97.68}=0.558, p=0.457$ & Not significant \\
\hline Young Male & 45.013 & 43.820 & $F_{1,44.00}=1.003, p=0.322$ & Not significant \\
\hline Young Female & 43.021 & 42.750 & $F_{1,44.00}=0.086, p=0.771$ & Not significant \\
\hline Middle Male & 42.640 & 43.218 & $F_{1,44.00}=0.289, p=0.594$ & Not significant \\
\hline Middle Female & 42.898 & 40.975 & $F_{1,39.00}=2.024, p=0.163$ & Not significant \\
\hline Older Male & 42.060 & 40.633 & $F_{1,39.00}=0.833, p=0.367$ & Not significant \\
\hline Older Female & 44.874 & 40.453 & $F_{1,25.69}=18.312, p<0.001$ & Significant \\
\hline
\end{tabular}

3

4 


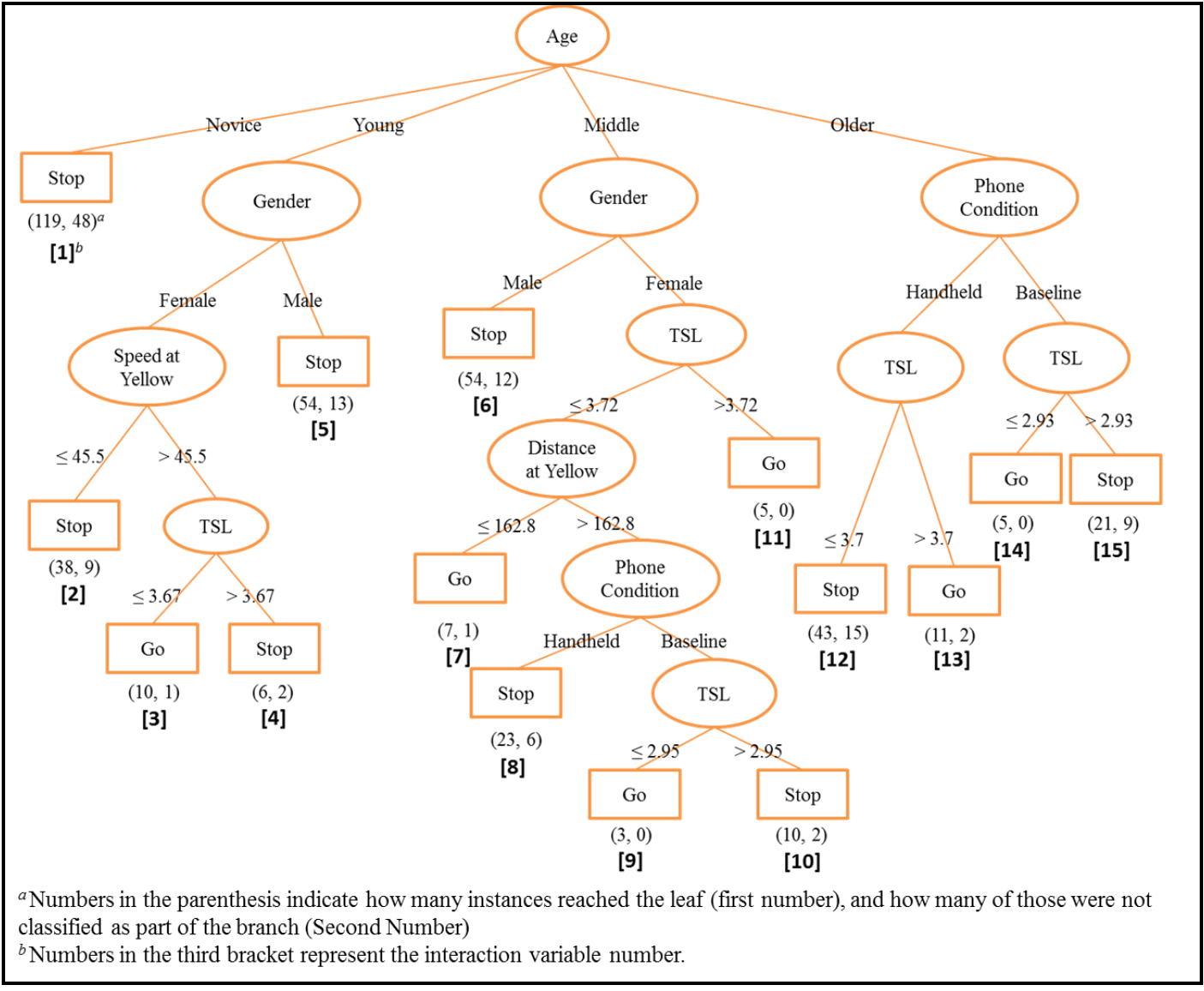

FIGURE 1 Decision tree diagram for the stop/go decision model 


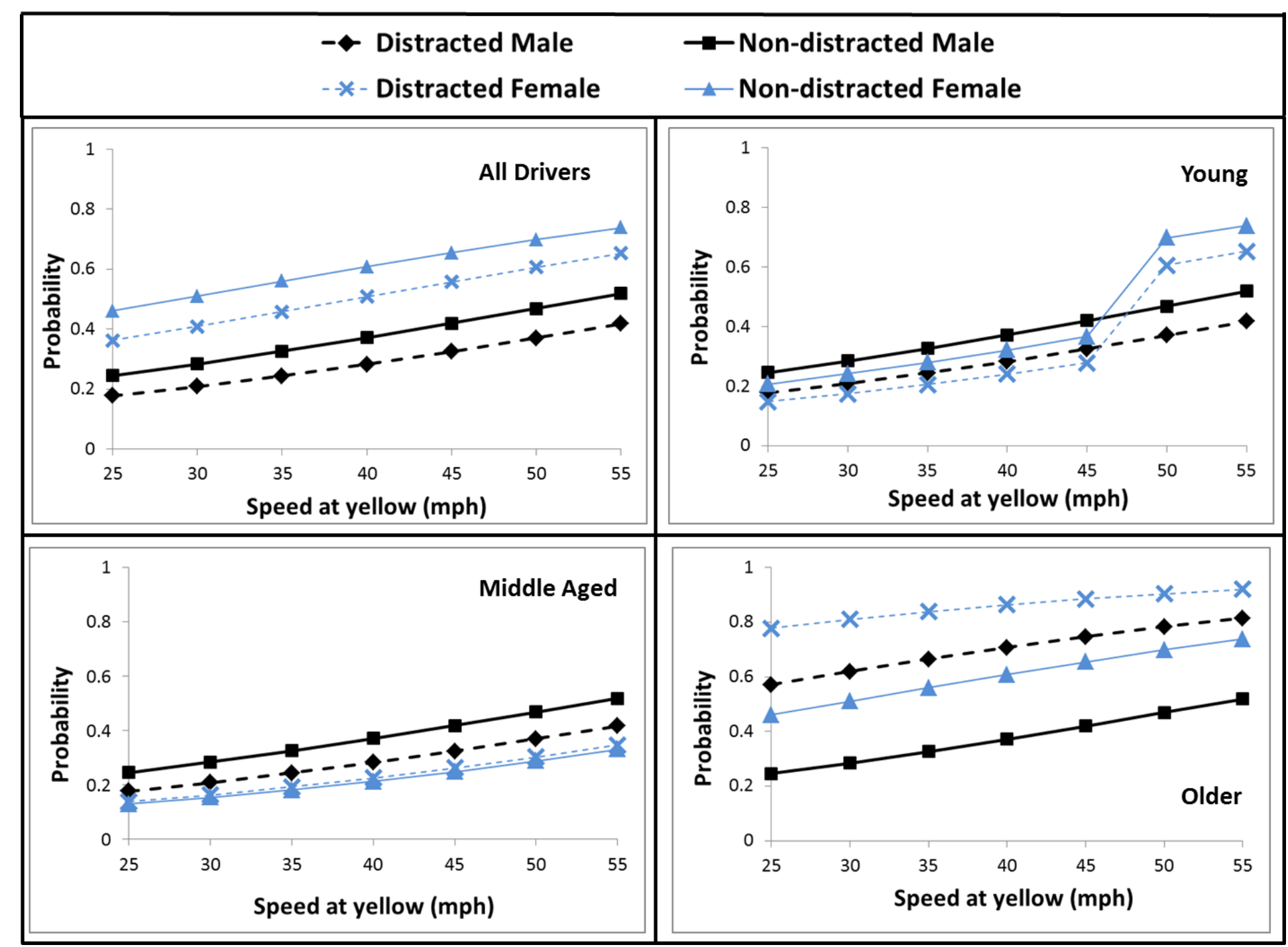

FIGURE 2 Probability of proceeding through yellow light for different driver groups 